University of Nebraska - Lincoln

DigitalCommons@University of Nebraska - Lincoln

2006

\title{
A Model for Predicting the Evolution of Multiple Cracks on Multiple Length Scales in Viscoelastic Composites
}

David H. Allen

University of Nebraska-Lincoln, dallen3@unl.edu

C. R. Searcy

Stress Engineering, Houston, TX

Follow this and additional works at: https://digitalcommons.unl.edu/engineeringmechanicsfacpub

Part of the Mechanical Engineering Commons

Allen, David H. and Searcy, C. R., "A Model for Predicting the Evolution of Multiple Cracks on Multiple Length Scales in Viscoelastic Composites" (2006). Faculty Publications from the Department of Engineering Mechanics. 60.

https://digitalcommons.unl.edu/engineeringmechanicsfacpub/60

This Article is brought to you for free and open access by the Mechanical \& Materials Engineering, Department of at DigitalCommons@University of Nebraska - Lincoln. It has been accepted for inclusion in Faculty Publications from the Department of Engineering Mechanics by an authorized administrator of DigitalCommons@University of Nebraska - Lincoln. 


\title{
A Model for Predicting the Evolution of Multiple Cracks on Multiple Length Scales in Viscoelastic Composites
}

\author{
D. H. Allen, College of Engineering, University of Nebraska-Lincoln, Lincoln, NE, USA \\ C. R. Searcy, Stress Engineering, Houston, TX, USA \\ Corresponding author - D. H. Allen, dhallen@unlnotes.unl.edu
}

\begin{abstract}
A model is presented herein for predicting the evolution of numerous cracks on multiple length scales, the objective of such a model being to develop the capability to predict failure of structural components to perform their intended tasks. Such a capability would then be useful as a predictive tool for designing structural components so as not to fail, but rather to succeed in performing their intended tasks. The model developed herein is somewhat involved, being based in continuum mechanics and thermodynamics, but is nevertheless expected to be cost effective (wherever sufficient accuracy permits) when compared to more costly experimental means of determining component life. An essential ingredient within the context of the model is that cracks must develop on widely differing length scales. Where this is observed to occur in nature, which is surprisingly often, there are potential simplifications over more generally described but practically untenable approaches, that can lead to (at least partly) computational multiscale algorithms capable of assimilating failure due to multiple cracking with a high degree of accuracy. The model presented herein will be briefly described within a mathematical framework, and an example problem will be presented that is representative of certain currently relevant technologies.
\end{abstract}

\section{Introduction}

When a crack is seen to extend in a solid, it is well known that a certain amount of energy is irreversibly lost from the object. Continued crack growth in a structural part is not indefinitely sustainable: component failure, normally due to a resulting instability, is the ultimate irrevocable end of this process. This is not a new concept, having been understood in some measure since ancient times. Indeed, the effects of fractures were discussed in technical terms by Da Vinci in the 16th century [1], as well as by Galileo [2] in the first scientific book on the mechanics of deformable bodies. However, the use of the mathematical concept of stress is less than two centuries old, and resulting theories capable of capturing the physics of fracture are little more than a century old. Perhaps the first great development in under- standing the mechanics of fracture is due to Griffith [3], who postulated that crack extension will occur in an object when

$$
G \geq G_{C}
$$

where $G$ is the energy released per unit area of crack produced, and $G_{C}$ is assumed to be a material constant. Within the context of modern day continuum mechanics, this allows one to include within the model of an initial boundary value problem a criterion for describing when the boundary, either internal or external, will change with time, and perhaps even how it will evolve.

As is now well known, Griffith's monumental proposal embodied by inequality (1) is possessed of some shortcomings. While it is quite adequate for some classes of solids, including many linear elastic ones, it is not accurate for others. Nevertheless, the concept is striking 
in simplicity, so much so that most models for improving on Griffith's criterion may in some way or another be said to be generalizations of Griffith's approach. Indeed, the second half of the 20th century is replete with an enormous body of literature intent upon improving our ability to predict crack growth in solids.

Progress notwithstanding, it would be presumptive to say that the ability to predict crack growth is completely resolved. Indeed, currently available models are still often shown to be quite inaccurate when compared to experimental data even for a single crack growing in a homogeneous solid. Oftentimes this shortcoming may be traced to the bulk material behavior of the solid in question. For example, in viscoelastic solids it is not uncommon to discover that while the general idea embodied in Griffith's model may still hold, the experimental data suggest that the critical energy release rate, $G_{C^{\prime}}$ is perhaps better represented as a material parameter depending on the loading rate or even the load history, rather than a material constant.

Furthermore, even for cases wherein substantive experimental data have demonstrated that the Griffith criterion is accurate for predicting the extension of a single crack in a solid, significant research remains to be done to show that the concept is accurate when the concept is applied to the prediction of the extension of multiple cracks, much less numerous cracks.

Nevertheless, we live in an age wherein structural parts are commonly designed and placed into service that are observed after component failure to have undergone the development of numerous cracks, often on widely different length scales. Examples of this phenomenon occur in such applications as virtually any and all aerospace structural composites, whether they be metallic or plastic (or both). Thus, carbon/epoxy laminates in wings, metal matrix composites in fan blades, glass mat thermoplastics, elevated temperature ceramic/ceramic composites - all are seen to develop unavoidable fields of cracks. And lest the reader has not checked out these applications lately, one can find an application much closer to every day life. Consider the roadway that we drive to work on every day, whether it be cementitious or asphaltic concrete. Rest assured that it is absolutely filled with cracks, on length scales running from microns to meters in scale. We live in the age of enlightenment: common sense demands that where such phenomena are observed, we should be able to predict them (the alternative, to avoid cracking, is not tenable, as it is apparent that in heterogeneous solids singular points cannot be avoided, so that if a fly lands on the part in its virgin state, cracks will grow!). Clearly, accurate models would create the potential for enormous cost savings to society, not to mention lives extended.

Thus it is that the state of research in solids has in the past decade turned to the prediction of the evolution of multiple cracks in structural parts. This is at least in part due to the development of computers with more and more speed and size. So perhaps we should just wait for the chip technology to simply "catch up". This argument can be laid to rest rather quickly in most cases by considering the following thought experiment. For a typical composite laminate, cracks can be seen in post mortem to number as many as tens of thousands per cubic meter. Conversely, computational models tell us that accurate simulation of a single crack extending in space and time requires thousands of finite elements, thus leading to a scenario that is computationally untenable were one to simply try the "brute force" approach of modeling every crack (on every length scale) at one time. Therefore, egotism must be tempered with a dose of reality: some error must be accommodated in order to obtain results. This then is at the heart of the problem to be described herein: to obtain sufficiently accurate results so that designers can use the modeling approach without requiring costs that exceed those encumbered with the experimental approach. It is hoped that the approach proposed herein is a step in that direction.

Before moving on to the modeling approach proposed herein, it is instructive to consider an alternative approach, partly because it is quite ingenious, but also because it actually leads the way to the methodology proposed herein. This alternative, sometimes called "continuum damage mechanics", perhaps grew out of research performed by Eshelby in the 1950s [4]. His interest was related to molecular scale voids such as dislocations, but the concept of homogenization utilized by him (as well as Hashin [5] and Hill [6] for undamaged media shortly thereafter) lent itself well to the idea that larger scale inclusions, including cracks, could somehow be incorporated into constitutive equations as something like an internal variable (or, in the case of phenomenological plasticity, plastic strain) when viewing the object at a distance far enough away that each crack cannot be seen. This approach gained widespread interest in the 1970's, and is even still actively pursued today. Perhaps one reason that such an approach is so inviting is that only a single scale analysis, that of the scale of the structural part, may be necessary to make predictions, and until recently computers were hard pressed to consider multiple scales simultaneously, especially in three dimensions. Unfortunately, the continuum damage mechanics approach is beset by several fundamental shortcomings that cannot be mitigated: (1) they require that complicated and expensive constitutive tests be performed on a length scale that is large enough to average out the effects of microcracks on the boundary of the constitutive specimen; (2) as a result of the incorporation of the damage into an evolving phenomenological parameter, the resulting constitutive equations are nonlinear; and (3) the averaging process at a length 
scale that is large compared to the length scale of the microcracks necessarily means that all physical parameters that exist on smaller length scales cannot be treated as design parameters unless further (exhaustive and expensive!) experimentation is pursued. For example, if one is attempting to model an asphaltic concrete roadway, the continuum damage mechanics approach can be applied to produce a nonlinear viscoelastic constitutive model (that includes a damage parameter accounting for microcracks). But if this approach is taken, such important design parameters as volume fractions of fines, aggregate size, and aggregate shape cannot be modeled without performing expensive and complicated constitutive experiments with various mixtures of each of these.

It is the last of these shortcomings of the continuum damage mechanics approach that is particularly troublesome, so much so that researchers have in the last decade sought more robust modeling approaches. Indeed, it is precisely the continuum damage mechanics approach that may have provided the spark necessary to ignite interest in a multiscaling approach to the problem. The necessity to perform so many experiments invariably has led some researchers to attempt to design "computational simulations" of the experiments needed to characterize the continuum damage models [7]. This of course requires that at the small scale required to perform simulations, one is led to the unavoidable conclusion that evolving internal boundaries must be incorporated into the simulation at this length scale. Furthermore, a representation of the constitutive behavior on the next larger length scale means that some sort of averaging process must be deployed in order to compare the computational simulation to the continuum damage model (or equivalently, the experiments utilized to derive it). This then supplies the starting point for a multiscale model that includes cracks on different length scales. The details of one such approach are reviewed in the following section.

\section{Analysis of the Object on the Microscale}

Consider an approach proposed herein that can be used on any number of length scales, $l_{\mu^{\prime}}$ observed in a solid object. The number of scales, $n$, utilized is determined by the physics of the problem on the one hand, and the amount of computational speed and size available on the other. To that end, consider a solid object with a region wherein microcracks are evolving on the smallest length scale considered, $l_{1}$, as shown in Figure 1. While it is not necessary (or even always correct) that a representative volume of the object on this length scale be accurately modeled by continuum mechanics, it will be assumed that this is the case in the current paper in order to simplify the discussion. Suppose that

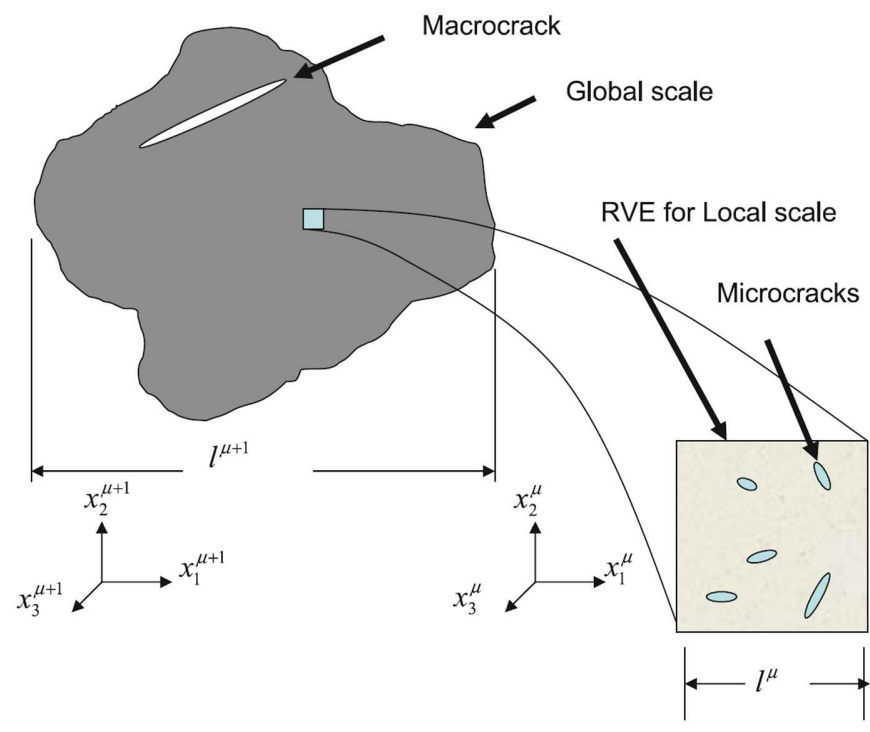

Figure 1. Two scale problem with cracks on both length scales.

the object can be treated as linear viscoelastic, again for simplicity, so that the following initial boundary value problem may be posed:

(a) conservation of linear momentum

$$
\vec{\nabla} \cdot \tilde{\sigma}_{\mu}+\rho \vec{f}=0 \quad \forall \vec{x}_{\mu} \in V_{\mu},
$$

where $\tilde{\sigma}_{\mu}$ is the Cauchy stress tensor defined on length scale $\mu, \rho$ is the mass density, and $\vec{f}$ is the body force vector per unit mass. Note that inertial effects have been neglected, implying that the length scale of interest is small compared to the next larger length scale, thus neglecting the effects of waves at this scale on the next scale up.

(b) strain-displacement equations

$$
\tilde{\varepsilon}_{\mu} \equiv \frac{1}{2}\left[\vec{\nabla} \vec{u}_{\mu}+\left(\vec{\nabla} \vec{u}_{\mu}\right)^{T}\right]
$$

where $\tilde{\varepsilon}_{\mu}$ is the strain tensor on the length scale $\mu$, and $\vec{u}$ ${ }_{\mu}$ is the displacement vector on the length scale $\mu$. Note that we have taken the linearized form of the strain tensor for simplicity, although a nonlinear form may be employed with out loss of generality.

(c) constitutive equations

$$
\tilde{\sigma}_{\mu}\left(\vec{x}_{\mu}, t\right)=\Omega_{\tau=-\infty}^{\tau=t}\left\{\tilde{\varepsilon}_{\mu}\left(\vec{x}_{\mu}, \tau\right)\right\},
$$

where $\vec{x}_{\mu}$ is coordinate location in the object on the length scale $\mu$, which has interior $V_{\mu}$ and boundary $\partial V_{\mu}$. The above description implies that the entire history of strain at any point in the body is mapped into the current stress, which is termed a viscoelastic material model. Because only the value of strain (the symmetric part of the deformation gradient is used in this model) is required at the point of interest, it is sometimes called a simple (or local) model [8]. Note that a local elastic material model, such as Hooke's law [9], is a special case of Equation (4). 
Equations (2)-(4) must apply in the body, together with appropriate initial and boundary conditions. These are then adjoined with a fracture criterion that is capable of predicting the growth of new or existing cracks anywhere in the object. There are multiple possibilities, but for example we could take the Griffith criterion given by inequality (1) above. The above then constitutes a well posed boundary value problem, albeit nonlinear due to the crack growth criterion (perhaps as well as the constitutive model (4)).

Solving this problem even for simple geometries is in itself a difficult challenge, as anyone who has every attempted to do so will attest. Nevertheless, assume that by some means (most likely computational) a solution can be obtained for the boundary conditions, geometry and precise form of constitutive Equation (4) at hand. Assume furthermore that the cracks that are predicted within the model dissipate so much energy locally that they may have further deleterious effects on the response at the next larger length scale. As an example, the so-called microcracks may in some way influence the development or extension of one or more macrocracks on the next larger length scale, $l_{2}$. It will be assumed that the cracks on the next larger length scale are much larger than those on the current scale, and that this restriction applies to all length scales for cracks in the object of interest:

$$
l_{\mu+1} \gg l_{\mu} \quad \mu=1, \cdots, n,
$$

where $n$ is the number of different length scales observed in the solid. Note that the above restriction is a necessary condition (but not sufficient) for the multiscale methodology proposed herein to produce reasonably accurate predictions on the larger length scale(s). If this condition is not satisfied, as in the case of a socalled localization problem, then there may indeed be no alternative to performing an exhaustive analysis at a single scale that takes into account all of the asperities simultaneously.

\section{Connecting the Microscale to the Macroscale}

In order to perform an analysis of the solid on the next length scale up from the local scale (termed the macroscale herein for simplicity), it is necessary to find a means of linking the state variables predicted on the local scale to those on the macroscale. Of course, the state variables at the local scale are predicted at an infinite collection of material points in the local domain $V_{\mu}+\partial V_{\mu^{\prime}}$ so that there is plenty of information available to supply to the next larger length scale. However, the objective herein is to find an efficient means of constructing this link without sacrificing too much accuracy. In other words, it is propitious to utilize the minimum data ob- tained at the local scale necessary to make a sufficiently accurate prediction at the macroscale. One way is to link the local scale to the macroscale via the use of mean fields. To see how this might work, consider the following mathematical expansion for the macroscale stress in terms of the local scale stress:

$$
\tilde{\sigma}_{\mu+1}=\overline{\tilde{\sigma}}_{\mu}+\sum_{j=1}^{\infty} \frac{1}{V_{\mu}|\overline{\vec{x}}|^{j}} \int_{V_{\mu}}\left(\tilde{\sigma}_{\mu}-\overline{\tilde{\sigma}}\right)|\vec{x}|^{j} \mathrm{~d} V,
$$

where

$$
\overline{\tilde{\sigma}}_{\mu} \equiv \frac{1}{V_{\mu}} \int_{V_{\mu}} \tilde{\sigma}_{\mu} \mathrm{d} V,
$$

is the volume averaged (or mean) stress at the local scale, and it is assumed that the local coordinate system is set at the geometric centroid of the local volume. Note that since the local domain $V_{\mu}+\partial V_{\mu}$ can be placed arbitrarily within the domain on the next larger length scale, $V_{\mu+1}$ $+\partial V_{\mu+1}$, the mean stress, $\overline{\tilde{\sigma}}_{\mu}$, is a continuously varying function of coordinates, $\vec{x}_{\mu+1}^{\mu}$, on the next larger length scale $\mu+1$, as shown in Figure 1. Note also that the terms within the summation in Equation (6) represent higher area moments of the stress tensor.

Now, it may be said without loss of generality that local scale conservation of momentum Equation (2) also applies to the global scale (assuming that quasi-static conditions still hold at this length scale):

$$
\vec{\nabla} \cdot \tilde{\sigma}_{\mu+1}+\rho \vec{f}=0 \quad \forall \vec{x}_{\mu+1} \in V_{\mu+1} .
$$

By using Equation (6), it can be shown that

$$
\lim _{l_{\mu} / l_{\mu+1} \rightarrow 0}\left(\tilde{\sigma}_{\mu+1}\right)=\overline{\tilde{\sigma}},
$$

and Equation (8) reduces to the following:

$$
\vec{\nabla} \cdot \overline{\tilde{\sigma}}_{\mu}+\rho \vec{f}=0 \quad \forall \vec{x}_{\mu+1} \in V_{\mu+1} .
$$

The similarity between Equations (2) and (10) is sufficiently striking that one is immediately tempted to use the same modeling algorithm on both length scales. This indeed is the approach that will be taken herein, but it must necessarily be said that Equation (10) is only exact in the limit, i.e., Equation (9) is a sufficient condition for Equation (10) to be exact. However, in all real circumstances Equation (9) cannot be satisfied, so that some error must necessarily be introduced by utilizing approximate Equation (10) in lieu of exact Equation (10).

The use of Equation (10) is termed herein a "mean field theory" because the higher order terms that are dropped from Equation (6) are essentially higher area moments of the local scale stress. Thus, the macroscale analysis is performed only in terms of the mean stress, 
$\overline{\tilde{\sigma}}$. Note that in cases wherein there is localization induced by damage or large strain gradients, one or more of the higher order terms will not be negligible. In this case, a mean field theory is no longer accurate, and a nonlocal approximation (including one or more of the higher order terms in Equation (6)), or even a full field analysis performed simultaneously on all length scales may be necessary in order to obtain reasonable accuracy. However, the necessity for converting to this procedure may be monitored by calculating the higher order terms in Equation (6) after each time step during the local scale analysis.

Now consider the standard deviation of the local scale stress, given by

$$
\tilde{\sigma}_{\mu}^{\mathrm{SD}} \equiv \frac{1}{V_{\mu}} \int_{V_{\mu}}\left(\tilde{\sigma}_{\mu}-\overline{\tilde{\sigma}}_{\mu}\right)^{2} \mathrm{~d} V .
$$

In an object in which the standard deviation of all of the state variables is small compared to their respective means is termed in this paper to "statistically homogeneous" (this, of course, implies that any singular points are bounded when integrated over the volume). It can also be shown that when Equation (9) is satisfied, the standard deviation of the local scale stress, given by Equation (11), goes to zero. Therefore, in many cases it is sufficient for the object to be statistically homogeneous at the local scale in order for Equation (10) to be an accurate representation at the macroscale. One implication of this result is that the microcracks contained within the local volume must be statistically homogeneous in location and orientation. If this is not the case, then higher order moments will necessarily have to be included at the macroscale [10].

Now note that, so long as any tractions on the crack faces are self equilibrating, Equation (2) may be used to show that [11-13]

$$
\overline{\tilde{\sigma}}_{\mu}=\frac{1}{V_{\mu}} \int_{\mu}\left(\tilde{\sigma}_{\mu} \cdot \vec{n}_{\mu}\right) \vec{x}_{\mu} \mathrm{d} S,
$$

where $\vec{n}_{\mu}$ is the unit outer normal vector on the local boundary, $\partial V_{\mu}$. Note that the boundary averaged stress given in Equation (12) actually is physically more palatable than the volume averaged stress given in Equation (7), as it is commensurate with the original definition of stress, as defined by Cauchy [26] to act on a surface.

The fact that the volume averaged stress is equivalent to the boundary averaged stress is of little importance when there are no cracks. However, when cracks grow and evolve with time, it becomes a very important aspect of the homogenization process, as will now be shown by considering the homogenization process for the strain tensor. It can be shown by careful employment of the divergence theorem that

$$
\overline{\tilde{\varepsilon}}_{\mu}=\tilde{\varepsilon}_{\mu+1}+\tilde{\alpha}_{\mu+1}
$$

where

$$
\overline{\tilde{\varepsilon}}_{\mu}=\frac{1}{V_{\mu}} \int_{V_{\mu}} \tilde{\varepsilon} \mathrm{d} V
$$

is the mean strain at the local scale

$$
\tilde{\varepsilon}_{\mu+1}=\frac{1}{V_{\mu}} \int_{\partial V_{\mu}^{E}} \frac{1}{2}\left[\vec{u}_{\mu} \vec{n}_{\mu}+\left(\vec{u}_{\mu} \vec{n}_{\mu}\right)^{T}\right] \mathrm{d} S,
$$

is the boundary averaged strain on the initial (external) boundary of the local volume, $\partial V_{\mu}{ }^{E}$, and

$$
\tilde{\alpha}_{\mu+1}=\frac{1}{V_{\mu}} \int_{\partial V_{\mu}^{I}} \frac{1}{2}\left[\vec{u}_{\mu} \vec{n}_{\mu}+\left(\vec{u}_{\mu} \vec{n}_{\mu}\right)^{T}\right] \mathrm{d} S,
$$

is the boundary averaged strain on the newly created (internal) boundary due to cracking, $\partial V_{\mu}{ }^{I}$, and is called a damage parameter $[4,14]$. Since kinematic Equation (15) is consistent with kinetic Equation (12), it is reasonable to construct constitutive equations at the macroscale in terms of these two variables, rather than in terms of volume averages. This is in striking contrast to the approach taken when there are no microcracks. In this case there is no difference between boundary averages and volume averages, as can be seen from the above equations. Nevertheless, using Equation (15) and the divergence theorem, it can be shown that

$$
\tilde{\varepsilon}_{\mu+1}=\frac{1}{2}\left[\vec{\nabla} \vec{u}_{\mu+1}+\left(\vec{\nabla} \vec{u}_{\mu+1}\right)^{T}\right],
$$

which can be seen to be similar in form to local Equation (3).

The construction of a homogenized macroscale initial boundary value problem, similar to that posed in Equations (2)-(4), is now nearly complete, as Equation (10) replaces Equation (2), and Equation (17) replaces Equation (3) at the macroscale. It remains to construct constitutive equations at the macroscale. Were one to utilize the continuum damage mechanics approach, it would be sufficient to simply postulate constitutive equations of the form:

$$
\tilde{\sigma}_{\mu+1}\left(\vec{x}_{\mu+1}, t\right)=\Omega_{\tau=-\infty}^{\tau=t}\left\{\tilde{\varepsilon}_{\mu+1}\left(\vec{x}_{\mu+1}, \tau\right), \alpha\left(\vec{x}_{\mu+1}, \tau\right)\right\} .
$$

The precise nature of this equation would then be determined by some curve fitting scheme either to experimental data provided from macroscale experiments, or 
the predictions made at the local scale. While this approach may be taken, as mentioned above, it removes the input parameters at the local scale from the design process. Therefore, it is preferable to take a multiscaling approach.

Instead, Equation (18) is obtained by direct substitution of local scale constitutive Equation (4) into the volume averaged stress Equation (7). The precise nature of the resulting equation will be depend on the choice of a constitutive model. As an example, consider the case wherein the local scale constitutive behavior is linear nonaging viscoelastic:

$$
\tilde{\sigma}_{\mu}\left(\vec{x}_{\mu}, t\right)=\int_{-\infty}^{t} \tilde{\tilde{E}}_{\mu}\left(\vec{x}_{\mu}, t-\tau\right) \frac{\partial \tilde{\varepsilon}_{\mu}\left(\vec{x}_{\mu}, \tau\right)}{\partial \tau} \mathrm{d} \tau,
$$

where $\tilde{\tilde{E}}\left(\vec{x}_{\mu^{\prime}}, t\right)$ is the relaxation modulus at the local scale. Direct substitution of Equation (19) into Equation (7), and subsequent careful utilization of Equations (6), (9), and (12) through (16) will result in a constitutive description at the macroscale that is of the following form [15]:

$$
\tilde{\sigma}_{\mu+1}\left(\vec{x}_{\mu+1}, t\right)=\int_{-\infty}^{t} \tilde{\tilde{E}}_{\mu+1}\left(\vec{x}_{\mu+1}, t-\tau\right) \frac{\partial \tilde{\varepsilon}_{\mu+1}}{\partial \tau} \mathrm{d} \tau,
$$

where

$$
\tilde{\tilde{E}}_{\mu+1}\left(\vec{x}_{\mu+1}, t\right) \equiv \frac{1}{V_{\mu}} \int_{V_{\mu}} \tilde{\tilde{E}}\left(\vec{x}_{\mu}, t-\tau\right) \mathrm{d} V,
$$

is the volume average of the relaxation modulus at the local scale, and is dependent on the damage incurred in the RVE at this scale, thereby implying that the material model described in Equation (20) is nonlinear.

It is now apparent that macroscale Equations (10), (13)-(16), and (19) correspond to local scale Equations (2)-(4), so that a similar algorithm may be utilized for the analysis on both scales. The significant difference is that the introduction of cracks at the local scale results in a more complex and inherently nonlinear formulation of the constitutive equations at the macroscale. This then completes the description of the homogenization process and the resulting macroscale initial boundary value problem.

\section{Fracture Model for Viscoelastic Media}

As mentioned in the introduction, there are several shortcomings of the Griffith criterion. First, it is often found to be inaccurate for viscoelastic media. Second, it is not convenient to utilize in a computational algorithm, which may be a necessary byproduct of modeling multiple cracks simultaneously. For these reasons, a different approach is taken herein for predicting crack growth in viscoelastic media. In the present paper, a cohesive zone model is utilized instead of the Griffith criterion. Models of this type are not new, having been introduced many years ago by Dugdale [16] and Barenblatt [17]. Initially at least, a primary motivation of these models was to account for ductility that occurs in many materials, a phenomenon that is not generally captured well by the Griffith criterion. Unfortunately, cohesive zone models suffer from several shortcomings that have inhibited their deployment until recently. These are essentially related to the inability to measure directly the material parameters necessary to characterize a particular cohesive zone model. Furthermore, a cohesive zone model is normally deployed in such a way that it is necessary to know where the crack will propagate a priori. For these reasons, cohesive zone models are only now finding widespread usage.

On the other hand, cohesive zone models are endowed with several significant strengths. Firstly, they are quite conveniently deployable into a finite element code by simply joining two or more subdomains with self equilibrating tractions, so that the domain may be treated as simply connected, and then allowing the tractions to relax to zero as a function of one or more observed state variables during problem solution, thereby resulting in the production of new surface area. Secondly, cohesive zone models can be formulated in such a way that they can more accurately capture fracture phenomena in some media than can the Griffith criterion. For example, it is often observed in viscoelastic media that the critical energy release rate required for crack extension is rate dependent.

Recently, Allen and Searcy $[18,19]$ have produced a cohesive zone model for some viscoelastic media that is formulated in such a way that the material parameters required to characterize the cohesive zone model can be obtained directly from microscale experiments. Furthermore, this model is inherently two scale in nature in that it utilizes the solution to a microscale scale continuum mechanics problem, together with a homogenization theorem to produce a cohesive zone model on the next larger length scale. The model has also been shown to be consistent with advanced fracture mechanics, in that the cohesive zone requires a nonstationary critical energy release rate in order for a crack to propagate [20-22].

This model will not be reviewed in detail herein since it has already been reported in the literature. However, a brief review is given here. As shown in Figure 2, the cohesive zone is postulated to be represented by a fibrillated or crazed zone that is small compared to the total cohesive zone area. The length scale of this IBVP is one length scale below that of the smallest local scale required in the multiscale problem. In this paper we term this the microscale, and arbitrarily assign the value $\mu=$ 1 to this length scale. The solution to this initial bound- 
Figure 2. Two scale problem showing a cohesive zone at the microscale.

\section{Local Scale}

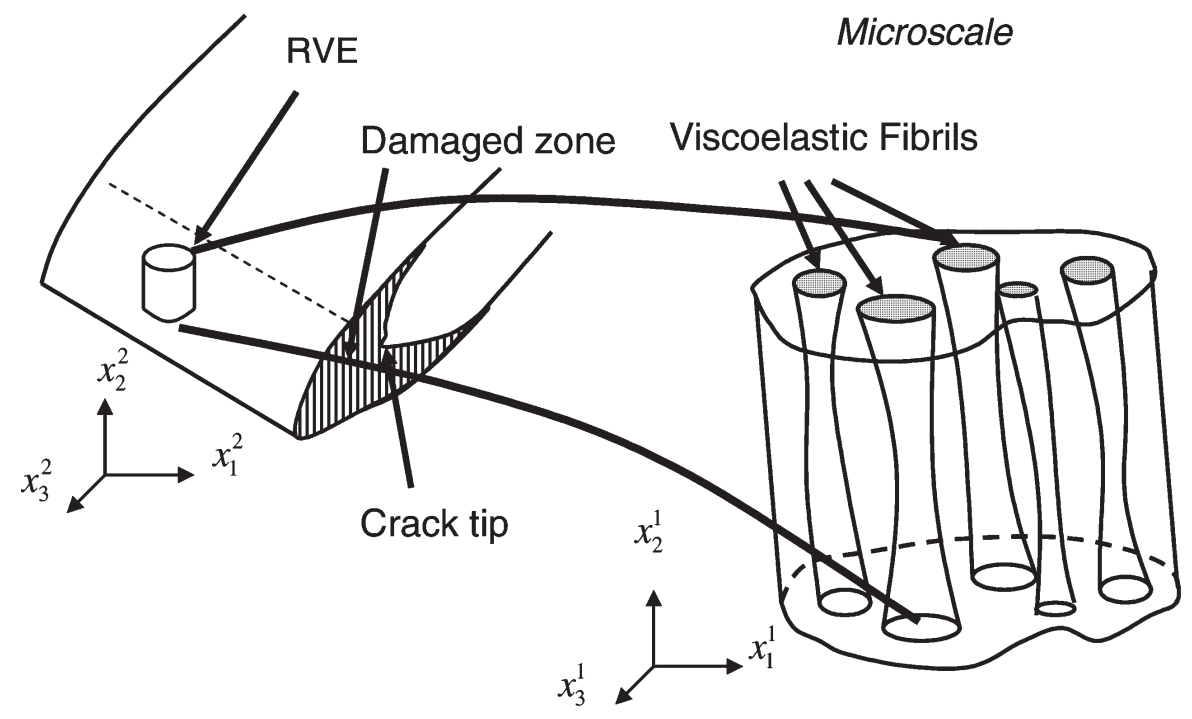

ary value problem (with geometry as shown in Figure 2, and governing equations identical to Equations (2)-(4)) has been obtained and homogenized, thus leading to the following traction-displacement relation in the cohesive zone [18]:

$$
\vec{T}=\frac{\vec{\delta}}{\delta_{0}}(1-\alpha) \int_{\tau=0}^{\tau=1} E(t-\tau) \frac{\partial \lambda}{\partial \tau} \mathrm{d} \tau \quad \forall \vec{x} \in \partial V_{1},
$$

where $E(t)$ is the uniaxial viscoelastic relaxation modulus of the undamaged cohesive zone material, $\partial V_{1}$ is the part of the boundary on which cohesive zones are active, $\delta$ is the crack opening displacement vector in the coordinate system, aligned with the crack faces, $\lambda$ is the Euclidean norm of the crack opening displacement vector, and $\alpha$ is the damage parameter, which in this case degenerates to a scalar, defined by

$$
\alpha \equiv \frac{A_{0}-\sum_{k=1}^{n f} A_{k}}{A_{0}},
$$

where $A_{0}$ is the undamaged planform cross-sectional area of a representative area of the cohesive zone, and $n f$ is the number of fibrils contained in the representative area. It can be seen that when all of the fibrils in a representative area fracture, the damage parameter a goes to unity and the traction vector in Equation (20) becomes zero, thereby inducing crack propagation. Note that the damage parameter, $\alpha$, does not exist on the smallest length scale. It appears as a natural byproduct of the homogenization process linking this scale to the next larger scale. This concept is not unlike the concept of temperature, which does not exist at the molecular scale, but arises as an outcome of kinetic motions averaged up to the continuum scale.
We should note herein that the damage parameter for this scale is a scalar, unlike that produced at the other length scales, as defined in Equation (16). This is due to the fact that for the case of a cohesive zone the homogenization process must be slightly altered to perform an area average rather than a volume average, as described in the previous section. In this case, the limit is taken as the dimension normal to the plane of the cohesive zone goes to zero, thereby, reducing the homogenized cohesive zone to a traction-displacement relation, rather than a stress-strain relation.

The next section describes how the cohesive zone model may be implemented to a multiscaling algorithm for performing analyses of structural components undergoing damage on multiple length scales.

\section{Formation of a Multiscale Algorithm}

The approach detailed above may be used to develop a multiscale algorithm for obtaining approximate solutions to problems containing multiple cracks growing simultaneously on widely differing length scales. This is accomplished by constructing a time stepping algorithm in which the global solution is first obtained for a small time step, assuming some initially damaged (or undamaged) state, as shown in Figure 3. The global solution for this time step is then utilized to obtain solutions for each integration point at the local scale, using the state variables obtained as output from the global analysis to obtain the solution at the local scale. The results for each integration point are then homogenized to produce the global constitutive equations to be used on the next time step at the global scale. This procedure is essentially an 


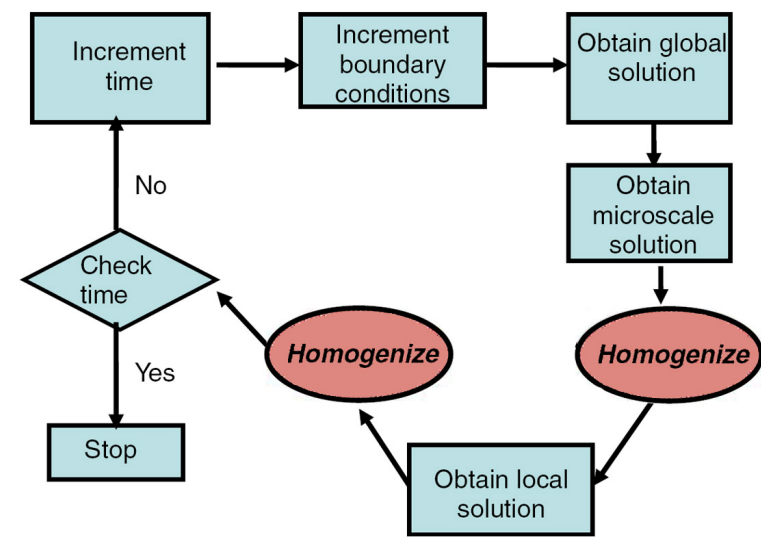

Figure 3. Flowchart showing multiscale computational algorithm.

operator splitting technique, assuming that there is one way coupling between the two length scales. Sufficient accuracy can usually be obtained by this method if successively smaller time steps are employed until convergence is obtained. Details of this approach may be found in Zocher and Allen [23] and Foulk et al. [24].

In principle, the approach described herein can be utilized on as many (continuum) length scales as necessary in order to solve complex problems. However, the limits of continuum scales in nature $\left(10^{-10} \mathrm{~m}<l<10^{3} \mathrm{~m}\right)$, and the requirement that the length scales be broadly separated, as given by inequality (5), lead one to the conclusion that only about five, or perhaps six length scales are physically possible. On the other hand, depending on the complexity of the given problem, only about three computational scales are practical with current computer capacities. Fortunately, there are few problems of current technological significance that require more than about three computational scales (there is generally no limitation on the number of analytic scales, as these require little computation, but analytic solutions, unlike the cohesive zone model described in the previous section, are not often attainable). The author and cowork- ers have been able to obtain solutions on a desktop computer by this technique using as many as four scales simultaneously (although it must be admitted that two of the scales were analytical) Phillips et al. [25]. For simplicity, a three scale problem is illustrated in Figure 4.

\section{An Example Problem}

Consider an example problem, as shown in Figure 5. The global object is an axisymmetric thick-walled pressure vessel subjected to an internal pressure. At the local scale the object is composed of unidirectional fibers imbedded in an isotropic matrix, as shown in the figure. The fibers are so small compared to the global scale that it is assumed to be sufficiently accurate to use a unit cell with periodic boundary conditions instead of an RVE, thereby producing improved computational efficiency at the local scale. Cracks may grow, but only at the interface between the fibers and matrix at the local scale. It is assumed that no cracks are present at the global scale. Figures 6, 7 show the evolution of cracking at three radial and three circumferential locations, respectively, in the analysis. Figure 8 shows a comparison of the predicted hoop stress as a function of radial location both with and without microscale cracking at the fiber-matrix interface. While this is simply a demonstrative problem, it shows the power of the multiscaling approach for structural design. For example, without recourse to further experimentation, the approach demonstrated here can be used to determine the fiber volume fraction that will produce the optimal structural configuration.

\section{Conclusion}

A multiscaling approach for modeling heterogeneous viscoelastic media, described herein, appears to be within reach of the design community. While it is only

\section{GLOBAL}

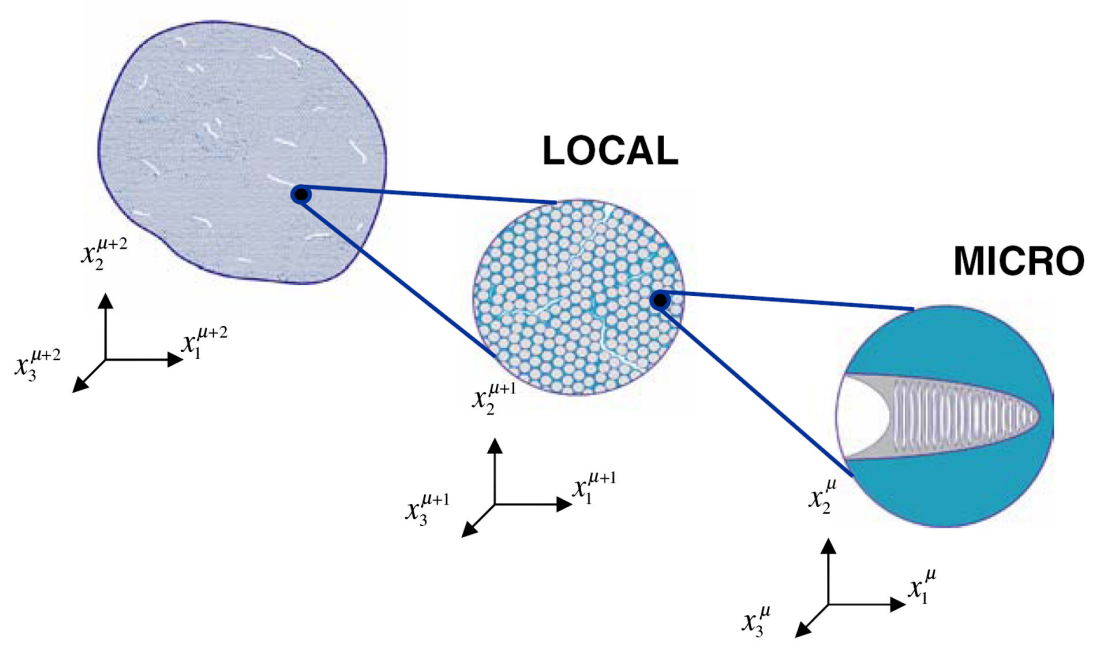

Figure 4. Example of three scale problem. 
Figure 5. Three scale axisymmetric cylinder problem.

\section{Global Geometry}

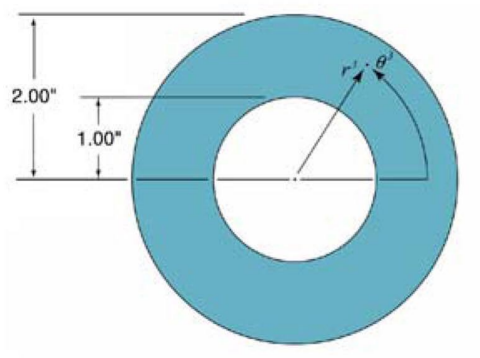

\section{Local Geometry}
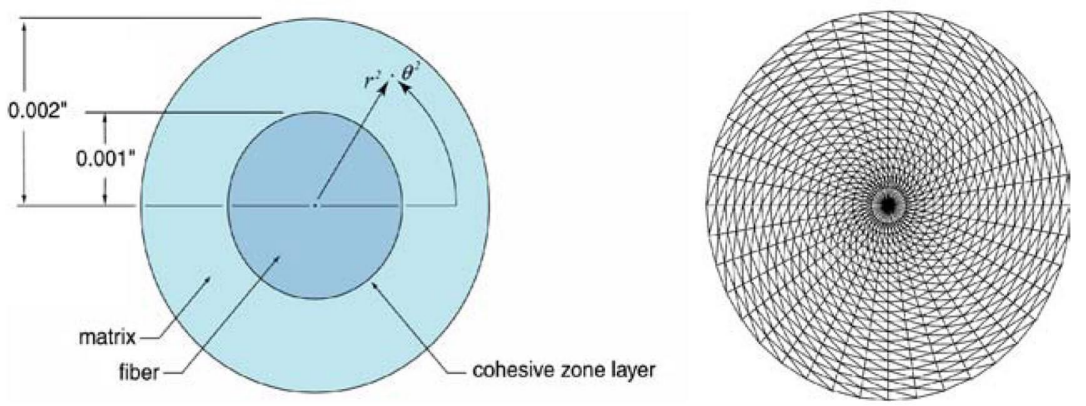

\section{Global Mesh}

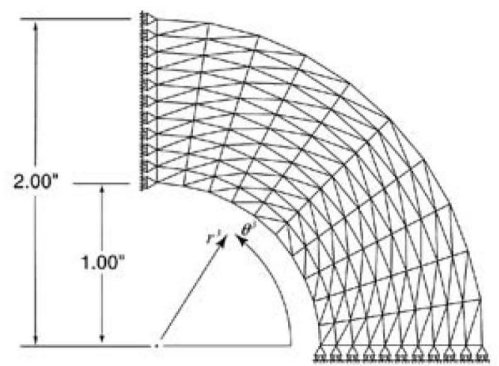

Local Mesh
Local Deformation vs. Global Radial Position

Figure 6. Predicted damage at three different radial positions in axisymmetric cylinder problem.

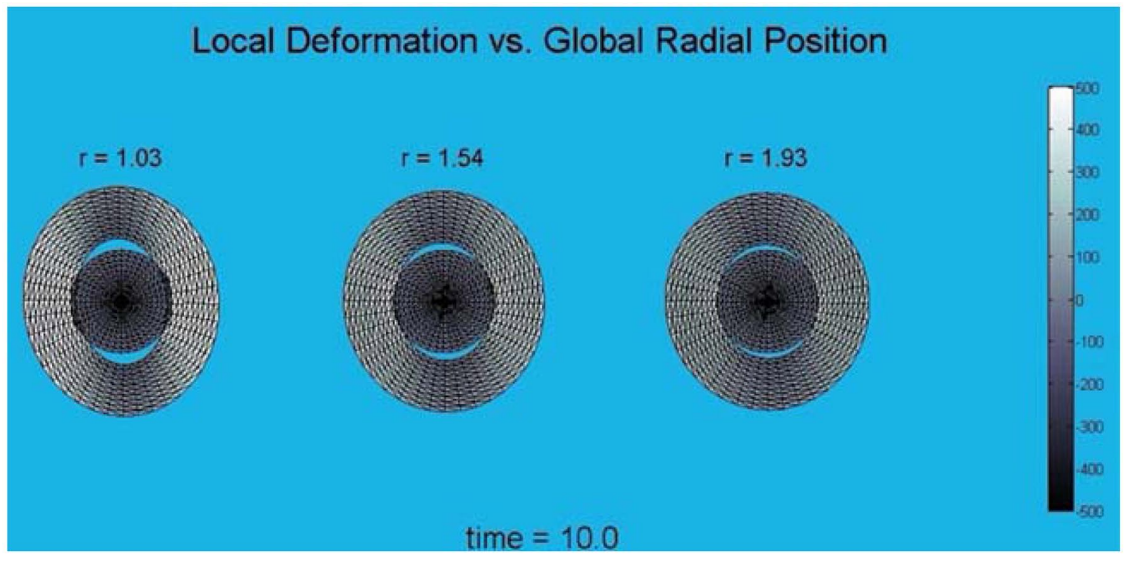

Local Deformation vs. Global Radial Position

Figure 7. Predicted damage at three different circumferential positions in axisymmetric cylinder problem.

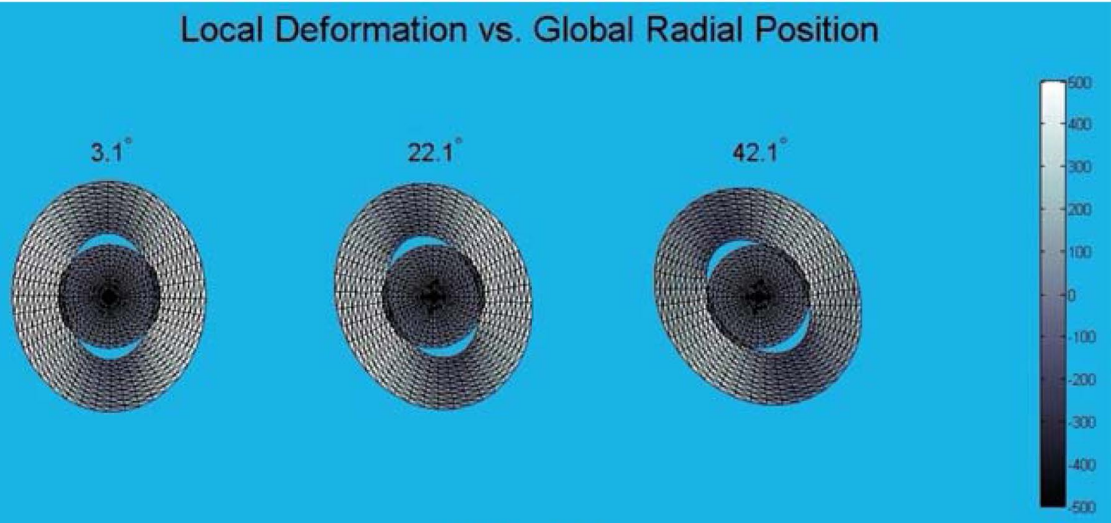




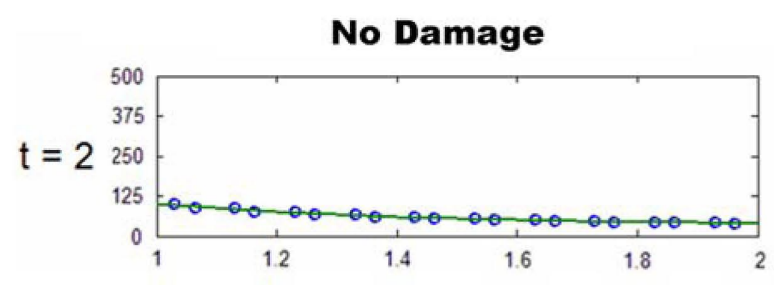

Damaged
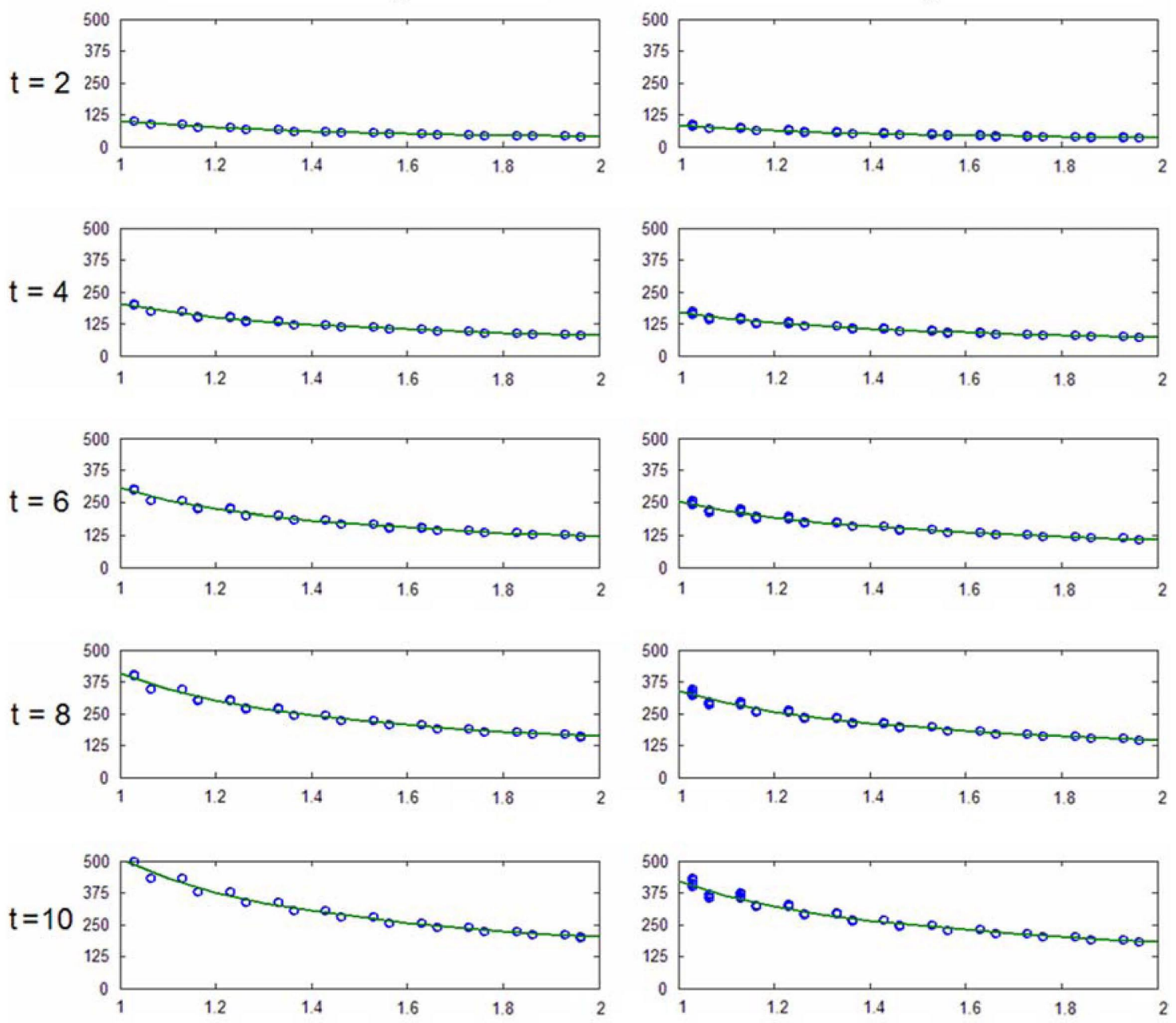

Figure 8. Hoop stress versus radial coordinate in axisymmetric cylinder problem.

in infancy, the results obtained herein indicate that it will not be long before such algorithms are in common use.

\section{Acknowledgments}

The authors are grateful for funding received for this research from the US Army Research Laboratory under contract no. W911NF-04-2-0011.

\section{References}

1. Da Vinci L (1970) In: Richter JP (ed) The notebooks of Leonardo Da Vinci

2. Galileo G (1636) Dialogues concerning two new sciences, Promethus Books

3. Griffith AA (1920) Philos Trans R Soc Lond A221:163

4. Eshelby JD (1957) Proc R Soc A421:376

5. Hashin Z (1964) Appl Mech Rev 17:1

6. Hill R (1965) J Mech Phys Solids 12:213

7. Allen DH (2002) Composites Sci Technol 61:2223

8. Day WA (1972) The thermodynamics of simple materials with fading memory, Springer Tracts in Natural Philosophy. Springer-Verlag, New York
9. TimoshenkoSP (1972) History of strength of materials. McGraw Hill 10. Allen DH (1994) In: Talreja R (ed) Damage mechanics of composite materials. Elsevier, pp 79-114

11. Boyd JG, Costanzo F, Allen DH (1993) Int J Damage Mech 2:209

12. Costanzo F, Boyd JG, Allen DH (1996) J Mech Phys Solids 44(3):333

13. Allen DH, Yoon C (1998) Int J Solids Struct 35:4035

14. Vakulenko AA, Kachanov ML (1971) Mekh Tver Tela 4:159

15. Searcy CR (2004) A multiscale model for predicting damage evolution in heterogeneous media. Ph.D. Thesis. Texas A\&M University

16. Dugdale DS (1960) J Mech Phys Solids 8:100

17. Barenblatt GI (1962) Adv Appl Mech 7:55

18. Allen DH, Searcy CR (2001a) Int J Fract 107:159

19. Allen DH, Searcy CR (2001b) Mech Mater 33:177

20. Costanzo F, Allen DH (1993) Int J Fract 63(1):27

21. Costanzo F, Allen DH (1996) Int J Eng Sci 33(15):2197

22. Yoon C, Allen DH (1999) Int J Fract 96:56

23. Zocher MA, Allen DH, Groves SE (1997) Int J Numer Methods Eng 40:2267

24. Foulk JW, Allen DH, Helms KLE (2000) Comput Methods Appl Mech Eng 183:51

25. Phillips ML, Yoon C, Allen DH (1999) J Eng Mater Technol 21:436

26. Cauchy A (1823) Bulletin de la Société Philomatique 9 